\title{
STUDY OF RENAL FUNCTION AFTER THROMBOLYTIC THERAPY IN PATIENTS WITH ACUTE MYOCARDIAL INFARCTION
}

\author{
Esam M. Mahfouz; K: faya S. Mohamed*, \\ Eman El-Safty anci Ashraf A. Omer
}

\author{
From \\ Internal Medicine (Cardiology Unit) \\ and Clinical Pathology* Departments.
}

\begin{abstract}
Thrombolytic therapy, the important therapeutic agents in patients with acute myocardial infarction has been reported to be associated with significant early onset protenuria.

The aim of this study was to determine whether streptokinase therapy is associated with changes in renal function (both glomerular and tubular) in excess of that expected in an acute vascular event; and the possible pathogenesis of any observed changes among patients with uncomplicated acute myocardial infarction.

Our work comprised 30 patients with uncomplicated acute myocardial infarction selected from coronary care unit Mansoura Emergency Hospital.
\end{abstract}

They comprised 15 patients who received streptokinase therapy (thrombolytic group) and 15 patients who did not receive streptokinase therapy (non thrombolytic group).

Laboratory tests reflecting both glomerular functions (serum createnine createnine clearance and urinary albumin excretion) and tubular functions (serum and urinary B2 microglobulin) were estimated for every patient on admission; 24 hours after initiation of therapy as well as on the 7 th day of therapy.

The results showed:

A) Non significant changes in renal functions among patients with acute myocardial infarction not receiving thrombolytic therapy.

MANSOURA MEDICAL JOURNAL 


\section{STUDY OF RENAL FUNCTION AFTER THROMBOLYTIC etc ...}

B) Thrombolytic therapy induced: (1) Non significant changes in serum createnine, createnine cleanance and serum B2 microglobulin; (2) transient glomerular impairment in the form of only increase in urinary albumin excretion and microalbuminuria that occurred $24 \mathrm{hr}$ after therapy and disappeared by the 7 th day and (3) mild renal tubular impairment in the form of significant increase in urinary $\mathrm{B} 2 \mathrm{mi}-$ croglobulin excretion that occurred 24 $\mathrm{hr}$ after therapy and persisted on the 7 th day.

The abnormal excretion of urinary albumin and B2-microglobulin were related to effective thrombolytic therapy and improved left ventricular performance.

The changes in the laboratory parameters after thrombolytic therapy were unrelated to presence or absence of associated diabetes mellitus, hypertension; mild renal impairment and dyslipidemia.

\section{Conclusion :}

Thrombolytic therapy can be used safely (unless there is other contraindication) among patients with diabetes mellitus, hypertension, dyslipidemia as well as with mild renal impairment.

Vol. 32, No. 1 \& 2 Jan. \& April, 2001

\section{INTRODUCTION}

Acute myocardial infarction is a serious medical emergency resulting in most cases from complete thrombotic occlusion of infarct-related coronary artery causing myocyte necrosis (1). Occlusion of a coronary artery leading to acute myocardial infarction appears to be the final common pathway resulting from a complex and dynamic interaction of coronary atherosclerosis, vasospasm and platelet activation ultimately leading to coronary artery thrombosis $(2,3,4)$.

In the presence of uncomplicated acute myocardial infarction, patients may show renal vasodilatation, and the elevation of atrial natriuretic peptides may play some role in this process(5). Both pre-renal azotemia and acute renal failure can complicate the marked reduction of cardiac output that occurs in cardiogenic shock(6). A physiologic compensation for this can occur by the increase in atrial natriuretic peptide, following acute myocardial infarction. The later increase is correlated with the degree of left ventricular failure (5).

Thrombolytic therapy is a traditional measure in treatment of acute myocardial infarction, which is believed to 
re-establish coronary blood flow (7). Streptokinase is a foreign protein and disadvantage to treatment with it is the occasional occurrance of immune reaction (8). Proteinuria may be a subclinical response that could be related to circulating specific antibody present at time of treatment (9).

Streptokinase was associated with significant early onset proteinuria of glomerular origin. This started to resolve by day 3 and without deterioration in overall renal function. The temporal relation to the initial fall in antibody titer suggests that it could be the result of immune complex deposition in the glomeruli (10).

The aim of this study was : (1) to determine wheather streptokinase therapy is associated with changes in renal function (both glomerular and tubular) in excess of that expected in an acute vascular event; (2) to determine the possible nature and etiology of any observed change and (3) the possible relation of any renal change to the presence of other concomitant diseases among patients with uncomplicated acute myocardial infarction.

\section{PATIENTS AND METHODS}

The present study comprised 30 patients with uncomplicated acute m:'ocardial infarction who were admitted and managed at coronary care unit Mansoura Emergency Hospital. They were 25 males and 5 females with ages ranging from 40 to 70 years $(57.5 \pm 8.7)$.

Patients were classified into two groups according to use of thrombolytic therapy:-

(A) Group receiving streptokinase which comprised 15 patients (the thrombolytic group).

(B) Group not receiving streptokinase (the non-thrombolytic group) which comprised 15 patients (and received only conventional th,erapy). Streptokinase was not given because of presence of any contraindication for its use.

Diagnosis of acute myocardial infarction was based on 2 of 3 criteria: (1) typical chest pain (2) definite electrocardiographic changes (ST elevation of at least $1 \mathrm{~mm}$ in 2 limb leads or $2 \mathrm{~mm}$ in 2 adjacent chest leads) and (3) rise in serum creatine phosphokinase (CPK) enzyme activity (11).

Based on clinical, electrocardiographic, echocardiographic and laboratory data, we excluded from our 
418 STUDY OF RENAL FUNCTION AFTER THROMBOLYTIC etc ...

study patients with : previous myocardial infarction, cardiogenic shock, congestive heart failure, collagen diseases, systemic vasculitis, urinary tract infection (pus cells $>5 / \mathrm{HPF}$ ), patients with initial microalbuminuria on admission, as well as patients with chronic renal disease or failure (createnine clearance $<30 \mathrm{ml} / \mathrm{min}$ ).

\section{All patients were subjected to} the following :

A- Thorough clinical evaluation and continuous monitoring and serial ECG recording.

B- Laboratory investigations :-

1) Cardiac enzymes:

- Creatinine phosphokinase (CPK) (on admission and after $24 \& 36$ hours) :

- Total CPK: By a three-step coupled enzyme system (12).

- CPK-MB isoenzyme: By immunoassay method (13).

- Lactic dehydrogenase (LDH) (on admission and after 24 \& 48 hours): By the biodynamic calometric procedure (14).

2) Some laboratory tests reflecting renal functions (three samples were taken: on admission, $24 \mathrm{hr}$ and 7 days after admission).

Vol. 32, No. 1 \& 2 Jan. \& April, 2001 a) Test reflecting glomerular function.

- Serum creatinine, urinary creatinine and estimation of creatinine clearance: By alkaline picrat method (15).

- Urine albumin level (16).

b) Test reflecting tubular function.

- Urine B2 microglobulin level: Enzymatic immunoassay (17).

- Serum B2 microglobulin level: Enzymatic immunoassay (17).

3) Complete urine analysis, and determination of total urinary protein (18).

4) Some liver function tests:

- Serum albumin: Calorometric determination (19).

- Serum bilirubin (20).

- Amino transferases (ALT \& AST): By Calirometric method (Transaminaseis kits) (21).

5) Complete blood picture.

6) Erythrocyte sedimentation rate (ESR).

7) Lipogram includes:

- Serum cholesterol: Enzymatic determination (22)

- Serum triglyceride: Enzymatic determination (23).

- HDL cholesterol and LDL cholesterol (24).

8) Fasting \& $2 \mathrm{hr}$ post-prandial 
blood sugar.

C. Plain X-ray chest PA view for, cardiomegaly, signs of lung congestion (25).

D- Echocardiographic examination (5-7 days after admission) for:

a) M-mode assessment of left ventricular dimensions and function via detection of end-systolic diameter, end-diastolic diameter, ejection fraction (EF), percent fractional shortening. (26)

b) Two dimensional analysis of segmental wall motions. (26)

E-Abdominal ultrasound.

The thrombolytic group was subdivided according to clinical and laboratory parameters as follow:

1- Presence of hypertension (9 patients hypertensive and 6 patients normotensive).

2- Presence of diabetes mellitus (7 patients diabetics and 8 patients not diabetics).

3- Createnine clearance ( 6 patients with creatinine clearance $>80 \mathrm{ml} /$ min and 9 patients with creatinine clearance $<80 \mathrm{ml} / \mathrm{min}$ ).

4- Serum cholesterol level (8 patients with cholesterol level >
$200 \mathrm{mg} / \mathrm{dl}$ and 7 patients with cholesterol level $\leq 200 \mathrm{mg} / \mathrm{dl}$ ).

\section{RESULTS}

1) The studied thrombolytic and non thrombolytic groups were well r.a:cried as regard age, blood pressure, blood glucose level, presence of hypertension, diabetes mellitus and site of infarction (table 1) and initral laboratory parameter (table 2).

2) Thrombolytic group showed significant increase in EF and \%FS than the non thrombolytic group reflecting better left ventricular performance (table 1).

3) The non thrombolytic group showed non significant serial changes in any test reflecting renal functions (table 3 \& Figur 1,2 ).

4) The whole thrombolytic group (tables 4 \& 5 \& Figure 1,3 ) and the different thrombolytic subgroups (tables 6-9) showed:-

- Absence of clinical proteinuria.

- Significantly increased urinary albumin excretion and increased incidence of microalbuminuria $24 \mathrm{hr}$ after thrombolytic therapy that returned to normal by the 7 th day of therapy $(P<$ 0.05).

- Significantly increased excretion of urinary B2-microalbumin and increased incidence of abnormal B2 uri- 
nary microglobumin level $24 \mathrm{hr}$ after thrombolytic therapy that persisted up to 7 th day of therapy $(P<0.05)$.

- Non significant serial changes in serum creatinine; creatinine clearance and serum $\mathrm{E}$ ?-microglobulin.

5) Non significant changes in all serial renal laboratory tests on comparing various thrombolytic subgroups:-

- Diabetics and non-diabetics (table 6).

- Hypertensives and nonhypertensives (table 7).

- Mild renal impairment and normal renal function (table 8).
- Dyslipidemics and nondys!:pidemics (table 9).

6) Significant positive correlation between: (1) left ventricular (LV) ejection fraction and urinary albumin level $(r=+0.540 ; P<0.05)$ and $(2)$ LV percent fibre shortening and urinary albumin level $(r=+0.637 ; P<0.05)$ (table 10).

7) Significant negative correlation between: (1) CPK and urinary B2 microglobulin ( $r=-0.565 ; P<0.05)$; (2) $\mathrm{LDH}$ and urinary albumin $(r=-0.509$; $P<0.05)$ and (3) LDH and urinary B2 microglobulin $(r=-0565 ; P<0.05)$ (table 10).

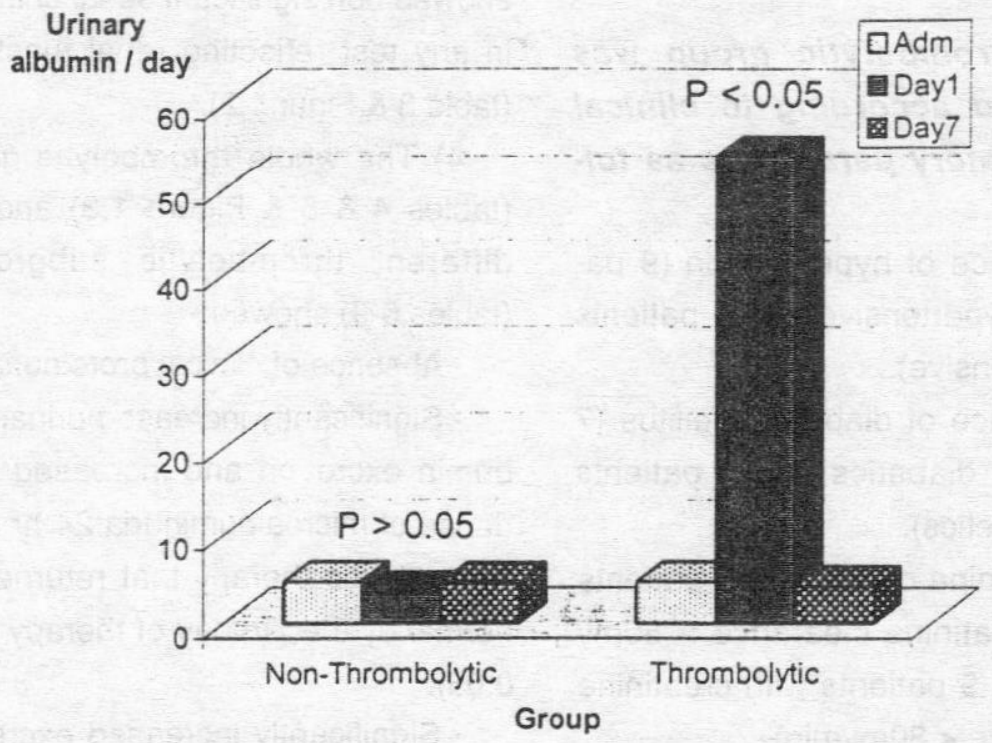

Figure (1): Urinary albumin excretion rate ( $\mu \mathrm{g} /$ day) among non-thrombolytic and thrombolytic groups.

Vol. 32, No. 1 \& 2 Jan. \& April, 2001 


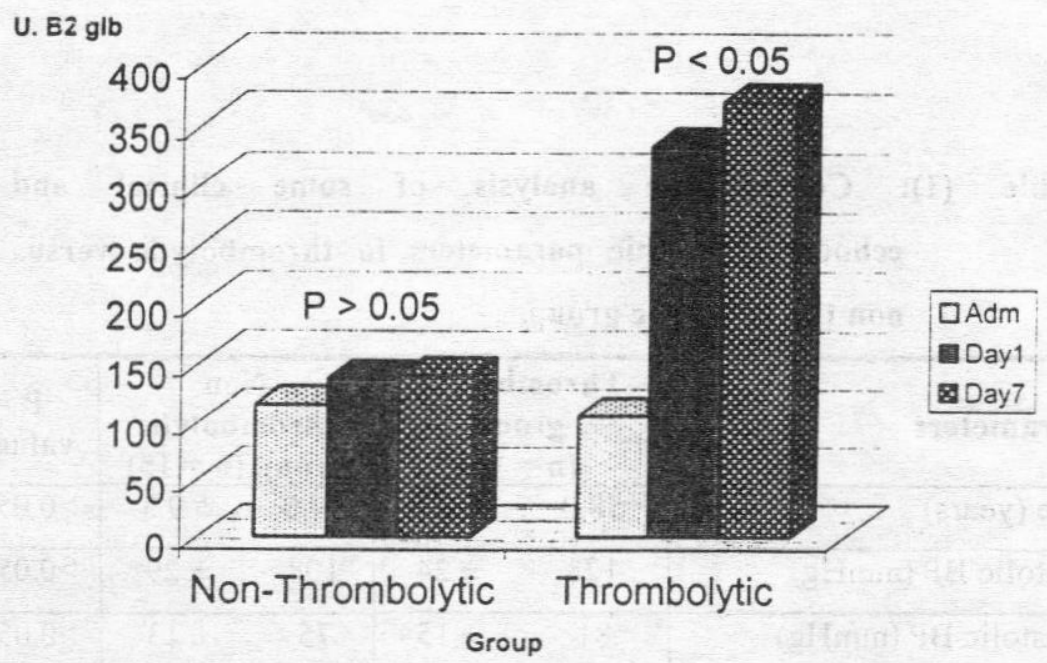

Figure (2) : Urinary B2-microglobulin excretion rate among the non-thrombolytic and thrombolytic groups.

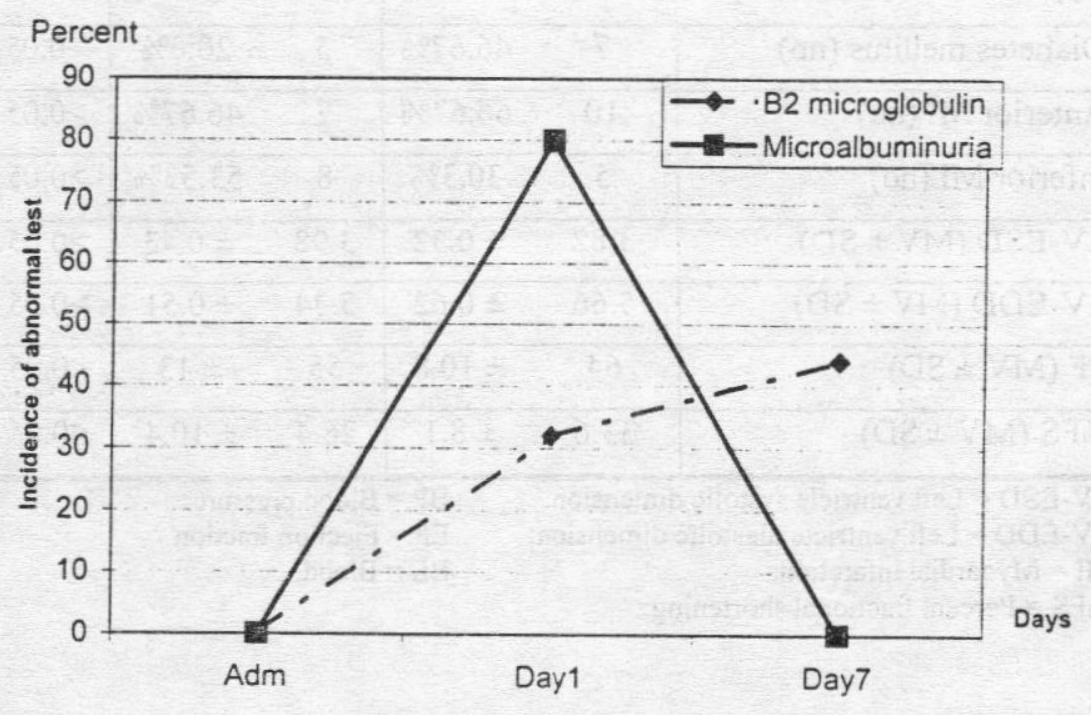

Figure (3): Serial incidence of microalbuminuria and abnormal B2-microglobulin among the thrombolytic group. 
Table (1): Comparative analysis of some clinical and echocardiographic parameters in thrombolytic versus non thrombolytic group.

\begin{tabular}{|c|c|c|c|c|c|}
\hline \multirow{2}{*}{$\begin{array}{l}\text { Parameters } \\
\text { Age (years) }\end{array}$} & \multicolumn{2}{|c|}{$\begin{array}{c}\text { Thrombolytic } \\
\text { group } \\
(\mathrm{n}=15)\end{array}$} & \multicolumn{2}{|c|}{$\begin{array}{c}\text { Non } \\
\text { thrombolytic } \\
\text { group }(\mathrm{n}=15)\end{array}$} & \multirow{2}{*}{$\begin{array}{c}\stackrel{P}{\text { value }} \\
>0.05\end{array}$} \\
\hline & 58.0 & \pm 8.8 & 57.0 & \pm 9.3 & \\
\hline Systolic BP (mmHg) & 128 & \pm 24 & 108 & \pm 29 & $>0.05$ \\
\hline Diastolic BP (mmHg) & 81 & \pm 15 & 75 & \pm 13 & $>0.05$ \\
\hline Fasting BL glucose (mg/dl) & 193 & \pm 24 & 181 & \pm 52 & $>0.05$ \\
\hline $2 \mathrm{hr}$ PP BL glucose (mg/dl) & 203 & \pm 65 & 195 & \pm 70 & $>0.05$ \\
\hline Hypertension (no) & 9 & $60 \%$ & 6 & $40 \%$ & $>0.05$ \\
\hline Diabetes mellitus (no) & 7 & $46.67 \%$ & 5 & $20.0 \%$ & $>0.05$ \\
\hline Anterior MI (no) & 10 & $66.67 \%$ & 7 & $46.67 \%$ & $>0.05$ \\
\hline Inferior MI (no) & 5 & $30.3 \%$ & 8 & $53.53 \%$ & $>0.05$ \\
\hline LV-ESD $(M V \pm S D)$ & 3.82 & \pm 0.32 & 3.98 & \pm 0.43 & $>0.05$ \\
\hline LV-EDD $(\mathrm{MV} \pm \mathrm{SD})$ & 5.66 & \pm 0.62 & 5.34 & \pm 0.51 & $>0.05$ \\
\hline $\mathrm{EF}(\mathrm{MV} \pm \mathrm{SD})$ & 64 & \pm 10.8 & 55 & \pm 13 & $<0.05$ \\
\hline$\% \mathrm{FS}(\mathrm{MV} \pm \mathrm{SD})$ & 33.6 & \pm 8.1 & 26.4 & \pm 10.4 & $<0.05$ \\
\hline \multicolumn{6}{|c|}{$\begin{array}{l}\text { LV-ESD = Left ventricle systolic dimension. } \\
\text { LV-EDD = Left ventricle diastolic dimension. } \\
\mathrm{MI}=\text { Myoardial infarction. } \\
\% \mathrm{FS}=\text { Percent fractional shortening. }\end{array}$} \\
\hline
\end{tabular}


Table (2): Comparative analysis of some laboratory parameters in thrombolytic versus non thrombolytic group (admission values).

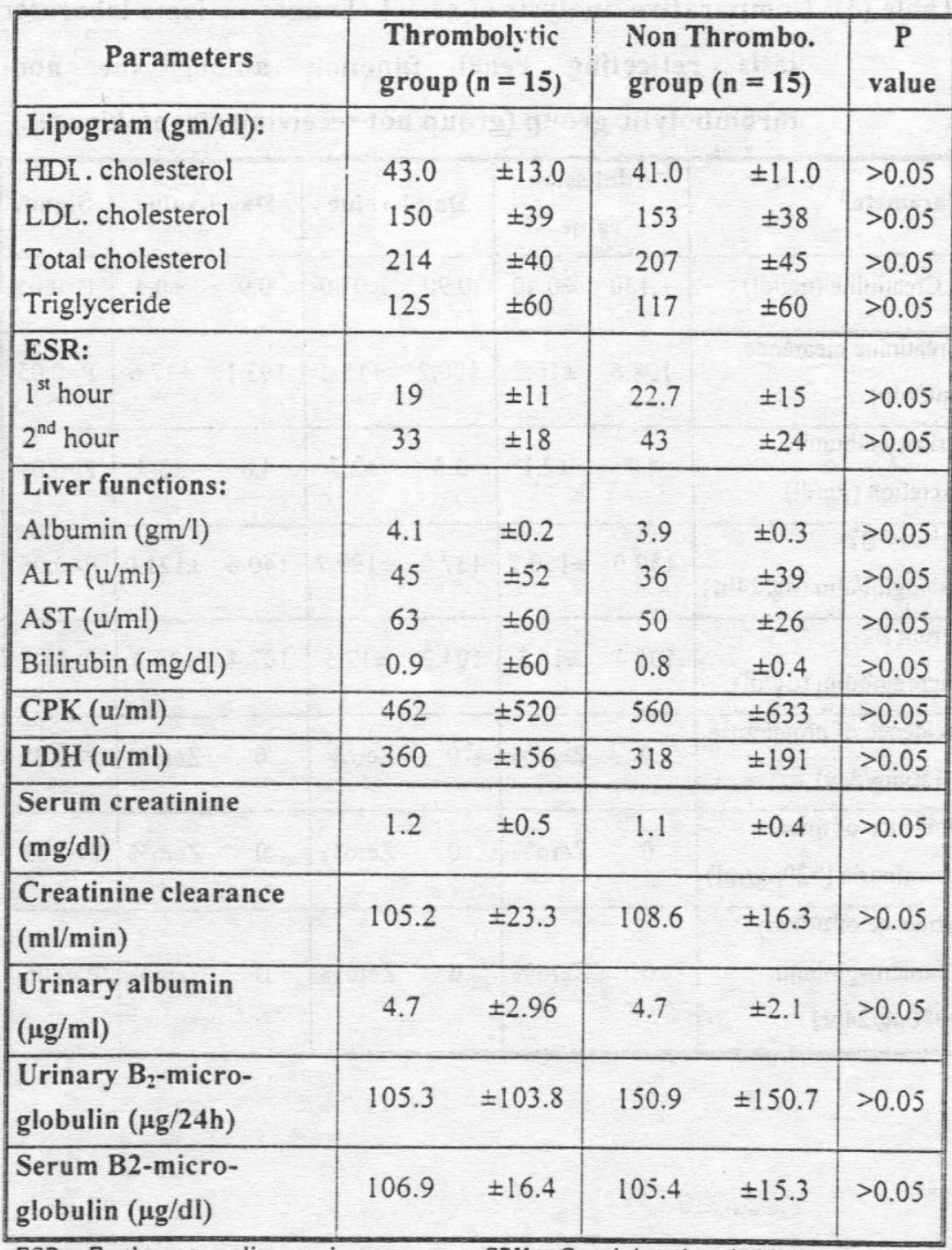

ESR $=$ Erythrocyte sedimentation rate. $\quad C P K=$ Creatinine phosphokinase.

$\mathrm{LDH}=$ Lactic dehydrogenase. 
Table (3): Comparative analysis of serial changes ir: some laborator tests reflecting renal function among the nonthrombolytic group (group not receiving streptokinase).

\begin{tabular}{|c|c|c|c|c|c|c|c|}
\hline Parameter & $\begin{array}{r}\text { Adm } \\
\text { va }\end{array}$ & $\begin{array}{l}\text { ission } \\
\text { lue }\end{array}$ & Day 1 & 1 value & Day 7 & 7 value & Signif. \\
\hline S.Creatinine (mg/dl) & 1.130 & \pm 0.40 & 0.90 & \pm 0.50 & 0.9 & \pm 0.4 & $P>0.05$ \\
\hline $\begin{array}{l}\text { Creatinine clearance } \\
(\mathrm{ml} / \mathrm{min})\end{array}$ & 108.6 & \pm 16.3 & 100.2 & \pm 15.2 & 103.1 & \pm 17.6 & $P>0.05$ \\
\hline $\begin{array}{l}\text { Urinary albumin } \\
\text { excretion }(\mu \mathrm{g} / \mathrm{dl})\end{array}$ & 4.7 & \pm 2.1 & 3.5 & \pm 2.7 & 4.6 & \pm 3.5 & $P>0.05$ \\
\hline $\begin{array}{l}\text { Urinary B2- } \\
\text { microglobulin }(\mu \mathrm{g} / 24 \mathrm{hr})\end{array}$ & 150.9 & \pm 150.7 & 137.0 & \pm 199.7 & 140.6 & \pm 121.0 & $P>0.05$ \\
\hline $\begin{array}{l}\text { Serum B2- } \\
\text { microglobulin }(\mu \mathrm{g} / \mathrm{dl})\end{array}$ & 105.4 & \pm 15.3 & 104.2 & \pm 17.6 & 107.4 & $=14.3$ & $P>0.05$ \\
\hline $\begin{array}{l}\text { Incidence of proteinuria } \\
\text { (>150mg/day) }\end{array}$ & 0 & Zero\% & 0 & Zero\% & 0 & Zero $\%$ & $P>0.05$ \\
\hline $\begin{array}{l}\text { Incidence of micro- } \\
\text { albuminuria }(>20 \mu \mathrm{g} / \mathrm{ml})\end{array}$ & 0 & Zero\% & 0 & Zero\% & 0 & Zero\% & $P>0.05$ \\
\hline $\begin{array}{l}\text { Incidence of urinary } \\
\text { B2-micro-globulin } \\
(>370 \mu \mathrm{g} / 24 \mathrm{hr})\end{array}$ & & Zero\% & 0 & Zero\% & 0 & Zero\% & $P>0.05$ \\
\hline
\end{tabular}


Table (4): Comparative analysis of serial changes in some parameters reflecting renal function amor.; :..te thrombolytic group (SK-subgroup).

\begin{tabular}{|c|c|c|c|c|c|c|}
\hline Parameter & $\begin{array}{l}\text { Admission } \\
\text { valve }\end{array}$ & Day 1 & 1 valve & Day 7 & valve & $\begin{array}{l}\text { Signif. } \\
\text { (F-test) }\end{array}$ \\
\hline S.Creatinine (mg/dl) & $1.2 \pm 0.5$ & 1.3 & \pm 0.9 & 1.2 & \pm 0.8 & $\mathrm{P}>0.05$ \\
\hline $\begin{array}{l}\text { Creatinine clearance } \\
(\mathrm{ml} / \mathrm{min})\end{array}$ & $105.2=23.3$ & 83.6 & $=17.4$ & 91.7 & \pm 18.6 & $P>0.05$ \\
\hline $\begin{array}{l}\text { Urinary albumin } \\
\text { excretion }(\mu \mathrm{g} / \mathrm{dl})\end{array}$ & $4.7 \pm 2.96$ & 53.6 & \pm 26.6 & 8.8 & \pm 4.2 & $P<0.05$ \\
\hline $\begin{array}{l}\text { Urinary B2- } \\
\text { microglobulin excretion } \\
(\mu \mathrm{g} / 24 \mathrm{hr})\end{array}$ & $105.3 \pm 103.8$ & 333.2 & \pm 405.6 & 370.2 & $=330.6$ & $P<0.05$ \\
\hline $\begin{array}{l}\text { Serum B2- } \\
\text { microglobulin (mg/dl) }\end{array}$ & $106.9 \pm 16.4$ & 104.9 & \pm 20.4 & 108.4 & $=14.5$ & $P>0.05$ \\
\hline $\begin{array}{l}\text { Incidence of proteinuria } \\
(>150 \mathrm{mg} / \text { day })\end{array}$ & $0 \quad$ Zero $\%$ & 0 & Zero\% & 0 & Zero $\%$ & $P>0.05$ \\
\hline $\begin{array}{l}\text { Incidence of micro- } \\
\text { albuminuria }(>20 \mu \mathrm{g} / \mathrm{ml})\end{array}$ & $0 \quad$ Zero\% & 12 & $80 \%$ & 0 & Zero \% & $P<0.05$ \\
\hline $\begin{array}{l}\text { Incidence of urinary } \\
\text { B2-micro-globulin } \\
(>370 \mu \mathrm{g} / 24 \mathrm{hr})\end{array}$ & $0 \quad$ Zero $\%$ & 5 & $32.33 \%$ & 7 & $\begin{array}{c}44.66 \\
\%\end{array}$ & $P<0.05$ \\
\hline
\end{tabular}


Table (5): Comparative analysis of incidence of mircoalbuminuria (> $20 \mathrm{ug} / \mathrm{min}$ ) and abnormal urinary B2-microglobulin excretion (> $370 \mathrm{ug} /$ day) among non thrombolytic and different thrombolytic subgroups.

\begin{tabular}{|c|c|c|c|c|c|c|}
\hline \multirow{3}{*}{ Studied group } & \multicolumn{3}{|c|}{ Microalbuminuria } & \multicolumn{3}{|c|}{$\begin{array}{c}\text { Abnormal urinary B2 } \\
\text { microglobulin }\end{array}$} \\
\hline & \multirow{2}{*}{\begin{tabular}{|ll|}
\multicolumn{2}{|l|}{ Adm. } \\
$\mathrm{n}$ & $\%$ \\
\end{tabular}} & Day 1 & \multirow{2}{*}{\begin{tabular}{|l|}
\multicolumn{2}{|l}{ Day 7} \\
$\mathrm{n} \quad \%$ \\
\end{tabular}} & \multirow{2}{*}{$\begin{array}{l}\text { Adm. } \\
\mathrm{n} \quad \% \\
\end{array}$} & Day 1 & Day 7 \\
\hline & & & & & n $\%$ & $\mathrm{n}$ \\
\hline $\begin{array}{l}\text { Non thrombolytic } \\
\text { group (15) }\end{array}$ & $0 \quad 0 \%$ & $0 \%$ & $\begin{array}{ll}0 & 0 \%\end{array}$ & $0 \quad 0 \%$ & $0 \%$ & $0 \%$ \\
\hline $\begin{array}{l}\text { Total thrombolytic } \\
\operatorname{gp}(15)\end{array}$ & $0 \quad 0 \%$ & $1280 \%$ & $00 \%$ & $0 \quad 0 \%$ & $33.3 \%$ & $7 \quad 44.6 \%$ \\
\hline Diabetic (7) & $00 \%$ & \begin{tabular}{|l|l}
6 & $85.7 \%$ \\
\end{tabular} & $\begin{array}{ll}0 & 0 \% \\
\end{array}$ & $0 \quad 0 \%$ & $28.6 \%$ & $3 \quad 42.9 \%$ \\
\hline \multirow[t]{2}{*}{ Non-diabetic (8) } & $00 \%$ & $6 \quad 87.5 \%$ & $0 \quad 0 \%$ & $0 \quad 0 \%$ & $37.5 \%$ & $50 \%$ \\
\hline & $P>0.05$ & $P>0.05$ & $P>0.05$ & $P>0.05$ & $P>0.05$ & $P>0.05$ \\
\hline Hypertensive (9) & $00 \%$ & $888.8 \%$ & $0 \quad 0 \%$ & $00 \%$ & $33.3 \%$ & $5 \quad 44.44 \%$ \\
\hline \multirow[t]{2}{*}{ Non-hypertens. (6) } & $0 \quad 0 \%$ & $466.6 \%$ & $\begin{array}{ll}0 & 0 \%\end{array}$ & $0 \quad 0 \%$ & $33.3 \%$ & $233.33 \%$ \\
\hline & $\mathrm{P}>0.05$ & $P>0.05$ & $P>0.05$ & $P>0.05$ & $P>0.05$ & $P>0.05$ \\
\hline Abnormal creat. (6) & $\begin{array}{ll}0 & 0 \% \\
\end{array}$ & \begin{tabular}{|ll}
5 & $83.3 \%$ \\
\end{tabular} & $\begin{array}{ll}0 & 0 \%\end{array}$ & $0 \%$ & $\begin{array}{|ll|}2 & 33.33 \% \\
\end{array}$ & $3 \quad 50.0 \%$ \\
\hline \multirow[t]{2}{*}{ Normal creat. (9) } & $\begin{array}{ll}0 & 0 \%\end{array}$ & $7 \quad 77.77 \%$ & $0 \quad 0 \%$ & $0 \quad 0 \%$ & $3 \quad 3.33 \%$ & $444.44 \%$ \\
\hline & $\mathrm{P}>0.05$ & $P>0.05$ & $P>0.05$ & $P>0.05$ & $P>0.05$ & $P>0.05$ \\
\hline Dyslipidemic (8) & $0 \quad 0 \%$ & $78 \%$ & $0 \quad 0 \%$ & $0 \%$ & $7.5 \%$ & $4 \quad 50.0 \%$ \\
\hline \multirow[t]{2}{*}{ Normal lipid (7) } & $\begin{array}{ll}0 & 0 \%\end{array}$ & $5 \quad 83 \%$ & $0 \quad 0 \%$ & $0 \quad 0 \%$ & $2 \quad 28.75 \%$ & $42.9 \%$ \\
\hline & $P>0.05$ & $P>0.05$ & $P>0.05$ & $P>0.05$ & $P>0.05$ & $P>0.05$ \\
\hline
\end{tabular}

Group number in between practice. 
Table (6): Comparative analysis of serial changes in some renal function test among the thrombolytic group (Diabetic versus non diabetic subgroups).

\begin{tabular}{|c|c|c|c|c|}
\hline Laboratory parameter & $\begin{array}{c}\text { Admission } \\
\text { valve } \\
\text { MV SD }\end{array}$ & $\begin{array}{c}\text { First day } \\
\text { valve } \\
\text { MV SD }\end{array}$ & $\begin{array}{l}\text { Day } 7 \text { valve } \\
\text { MV SD }\end{array}$ & $\begin{array}{c}\text { Significance } \\
\text { (F-test) }\end{array}$ \\
\hline \multicolumn{5}{|l|}{ S.creatinine } \\
\hline Diabetic gp & $1.50 \pm 0.616$ & $1.56 \pm 0.960$ & $1.60 \pm 0.698$ & $P>0.05$ \\
\hline \multirow[t]{2}{*}{ Non-diabetic gp } & $1.09 \pm 0.24$ & $1.15 \pm 0.611$ & $1.09 \pm 0.748$ & $P>0.05$ \\
\hline & $P>0.05$ & $P>0.05$ & $P>0.05$ & \\
\hline \multicolumn{5}{|l|}{ Cr. clearance } \\
\hline Diabetic gp & $96.30 \pm 17.80$ & $78.7 \pm 13.8$ & $84.70 \pm 21.17$ & $P>0.05$ \\
\hline \multirow[t]{2}{*}{ Non-diabetic gp } & $112.8 \pm 23.20$ & $86.5 \pm 18.8$ & $97.07 \pm 14.30$ & $P>0.05$ \\
\hline & $P>0.05$ & $P>0.05$ & $P>0.05$ & \\
\hline \multicolumn{5}{|l|}{ Serum $B_{2}$ microglob. } \\
\hline Diabetic gp & $109.0=16.2$ & $108.0 \pm 18.3$ & $108.6 \pm 14.7$ & $P>0.05$ \\
\hline \multirow[t]{2}{*}{ Non-diabetic gp } & $108.0 \pm 15.0$ & $107.0 \pm 16.8$ & $106.0 \pm 15.0$ & $P>0.05$ \\
\hline & $P>0.05$ & $\mathrm{P}>0.05$ & $P>0.05$ & \\
\hline \multicolumn{5}{|l|}{ Urinary albumin } \\
\hline Diabetic gp & $5.02 \pm 2.40$ & $62.60 \pm 20.30$ & $9.03 \pm 3.60$ & $P<0.05$ \\
\hline \multirow[t]{2}{*}{ Non-diabetic gp } & $4.40 \pm 3.50$ & $46.80 \pm 28.40$ & $6.65 \pm 2.30$ & $P<0.05$ \\
\hline & $P>0.05$ & $P>0.05$ & $P>0.05$ & \\
\hline \multicolumn{5}{|l|}{ Urinary $\mathrm{B}_{2}$ microglobulin } \\
\hline Diabetic gp & $98.60 \pm 120.6$ & $226.1 \pm 294.2$ & $390 \pm 366.0$ & $P<0.05$ \\
\hline \multirow[t]{2}{*}{ Non-diabetic gp } & $112.2 \pm 99.8$ & $376.2 \pm 393.4$ & $318 \pm 209.8$ & $P<0.05$ \\
\hline & $P>0.05$ & $P>0.05$ & & \\
\hline
\end{tabular}

Diabetic group comprised 7 patients.

Non-diabetic group comprised 8 patients. 
Table (7): Comparative analysis of serial changes in some renal function test among the thrombolytic group (Hypertensive versus non hypertensive subgroups).

\begin{tabular}{|c|c|c|c|c|}
\hline Laboratory parameter & $\begin{array}{l}\text { Admission } \\
\text { valve } \\
\text { MV SD }\end{array}$ & \begin{tabular}{|c|} 
First day valve \\
MV SD
\end{tabular} & $\begin{array}{l}\text { Day } 7 \text { valve } \\
\text { MV SD }\end{array}$ & $\begin{array}{l}\text { Signifi- } \\
\text { cance } \\
\text { (F-test) }\end{array}$ \\
\hline $\begin{array}{l}\text { S.creatinine } \\
\text { Hypertensive gp } \\
\text { Non-hypertensive gp }\end{array}$ & $\begin{array}{c}1.44 \pm 0.753 \\
0.95 \pm 0.607 \\
P>0.05\end{array}$ & $\begin{array}{c}1.5=0.986 \\
1.10=0.420 \\
P>0.05\end{array}$ & $\begin{array}{l}1.39 \pm 0.680 \\
0.95 \pm 0.63\end{array}$ & $\begin{array}{l}P>0.05 \\
P>0.05\end{array}$ \\
\hline $\begin{array}{l}\text { Cr. clearance } \\
\text { Hypertensive gp } \\
\text { Non-hypertensive gp }\end{array}$ & $\begin{array}{c}101.22 \pm 24.36 \\
110.67 \pm 20.14 \\
P>0.05\end{array}$ & $\begin{array}{c}79.84=17.24 \\
90.35=15.05 \\
P>0.05\end{array}$ & $\begin{array}{c}85.11 \pm 20.40 \\
95.60=18.12 \\
P>0.05\end{array}$ & $\begin{array}{l}P>0.05 \\
P>0.05\end{array}$ \\
\hline $\begin{array}{l}\text { Serum } B_{2} \text { microglob. } \\
\text { Hypertensive gp } \\
\text { Non-hypertensive gp }\end{array}$ & $\begin{array}{c}98.67=25.49 \\
106.5=22.06 \\
P>0.05\end{array}$ & $\begin{aligned} 103.89 & =18.67 \\
106.5 & =16.29 \\
P> & 0.05\end{aligned}$ & $\begin{array}{c}115.36=11.75 \\
104.0=16.79 \\
P>0.05\end{array}$ & $\begin{array}{l}P>0.05 \\
P>0.05\end{array}$ \\
\hline $\begin{array}{l}\text { Urinary albumin } \\
\text { Hypertensive gp } \\
\text { Non-hypertensive gp }\end{array}$ & $\begin{array}{c}4.49 \pm 2.48 \\
4.06 \pm 3.82 \\
P>0.05\end{array}$ & $\begin{array}{c}62.22 \pm 19.01 \\
40.67 \\
P>20.47 \\
P>0.05\end{array}$ & $\begin{aligned} 9.02 & \pm 4.29 \\
8.50 & =4.46 \\
P> & 0.05\end{aligned}$ & $\begin{array}{l}P<0.05 \\
P<0.05\end{array}$ \\
\hline $\begin{array}{l}\text { Urinary } \mathrm{B}_{2} \text { microglobulin } \\
\text { Hypertensive gp } \\
\text { Non-'yypertensive gp }\end{array}$ & $\begin{array}{c}110.33 \pm 109.8 \\
101.50 \pm 94.2 \\
P>0.05\end{array}$ & $\begin{array}{c}296.44 \pm 235.43 \\
38.40=418.0 \\
P>0.05\end{array}$ & $\begin{array}{c}329.0=343.2 \\
485.0=371.0 \\
P>0.05\end{array}$ & $\begin{array}{l}P<0.05 \\
P<0.05\end{array}$ \\
\hline
\end{tabular}

Hypertensive group comprised 9 patients.

Non-hypertensive group comprised 6 patients. 
Table (8): Comparative analysis of serial changes in some renal function test among the thrombolytic group (mild renal impairment versus normal renal subgroups).

\begin{tabular}{|c|c|c|c|c|}
\hline \multirow[t]{2}{*}{ Laboratory parameter } & $\begin{array}{c}\text { Admission } \\
\text { valve }\end{array}$ & $\begin{array}{c}\text { First day } \\
\text { valve }\end{array}$ & Day 7 valve & \multirow{2}{*}{$\begin{array}{l}\text { Signif- } \\
\text { icance } \\
\text { (F-test) }\end{array}$} \\
\hline & MV SD & MV SD & MV $3 D$ & \\
\hline \multicolumn{5}{|l|}{ S.creatinine } \\
\hline Mild renal impairment $\mathrm{gp}$ & $1.62 \pm 0.509$ & $2.03 \pm 0.98$ & $1.48 \pm 0.568$ & $P>0.05$ \\
\hline \multirow[t]{2}{*}{ Normal renal gp } & $0.944 \pm 0.150$ & $1.024=0.402$ & $1.04 \pm 0.398$ & $P>0.05$ \\
\hline & $\mathrm{P}<0.05$ & $\mathrm{P}<0.05$ & $P<0.05$ & \\
\hline \multicolumn{5}{|l|}{ Cr. clearance } \\
\hline Mild renal impairment gp & $\begin{array}{ll}72.83 & 27.80\end{array}$ & $60.3 \pm 21.9$ & $68.7 \pm 17.2$ & $P>0.05$ \\
\hline \multirow[t]{2}{*}{ Normal renal gp } & $\begin{array}{ll}119.7 & 18.58\end{array}$ & $93.7 \pm 19.5$ & $99.7 \pm 16.2$ & $P>0.05$ \\
\hline & $P<0.05$ & $P<0.05$ & $P<0.05$ & \\
\hline \multicolumn{5}{|l|}{ Serum $B_{2}$ microglob. } \\
\hline Mild renal impairment gp & $109.0 \quad 16.0$ & $102.33 \pm 19.25$ & $105.67=65.0$ & $P>0.05$ \\
\hline \multirow[t]{2}{*}{ Normal renal gp } & 110.4411 .84 & $108.44 \pm 21.85$ & $110.44=17.4$ & $P>0.05$ \\
\hline & $P>0.05$ & $P>0.05$ & $P>0.05$ & \\
\hline \multicolumn{5}{|l|}{ Urinary albumin } \\
\hline Mild renal impairment gp & $5.20 \quad 2.50$ & $71.10 \pm 23.2$ & $9.4=3.9$ & $P<0.05$ \\
\hline \multirow[t]{2}{*}{ Normal renal gp } & $4.20 \quad 3.30$ & $41.9 \pm 28.9$ & $8.4 \pm 6.8$ & $P<0.05$ \\
\hline & $P>0.05$ & $P>0.05$ & $P>0.05$ & \\
\hline \multicolumn{5}{|l|}{ Urinary $B_{2}$ microglobulin } \\
\hline Mild renal impairment gp & $120.7 \quad 136.6$ & $291.1=284.0$ & $371.3 \pm 290.1$ & $P<0.05$ \\
\hline \multirow[t]{2}{*}{ Normal renal gp } & 95.183 .0 & $387.0 \pm 446.2$ & $393.0 \pm 361.8$ & $P<0.05$ \\
\hline & $P>0.05$ & & $P>0.05$ & \\
\hline
\end{tabular}

Mild renal impairment group comprised 6 patients.

Normal renal group comprised 9 patients. 
Table (9): Comparative analysis of serial changes in some renal function test among the thrombolytic group (Dyslepidemia versus non dyslepidemia subgroups).

\begin{tabular}{|c|c|c|c|c|}
\hline \multirow[t]{2}{*}{ Laboratory parameter } & $\begin{array}{l}\text { Admission } \\
\text { valve }\end{array}$ & $\begin{array}{c}\text { First day } \\
\text { valve }\end{array}$ & Day 7 valve & \multirow{2}{*}{$\begin{array}{c}\text { Signifi- } \\
\text { cance } \\
\text { (F-test) }\end{array}$} \\
\hline & MV SD & MV SD & MV SD & \\
\hline \multicolumn{5}{|l|}{ S.creatinine } \\
\hline Dyslepidemic gp & $1.42 \pm 0.57$ & $1.58 \pm 0.980$ & $1.20 \pm 0.67$ & $P>0.05$ \\
\hline \multirow[t]{2}{*}{ Normal lipid gp } & $1.02 \pm 0.36$ & $1.08 \pm 0.69$ & $1.04 \pm 0.84$ & $P>0.05$ \\
\hline & $P>0.05$ & $P>0.05$ & $P>0.05$ & \\
\hline \multicolumn{5}{|l|}{ Cr. clearance } \\
\hline Dyslepidemic gp & $100.4 \pm 23.9$ & $86.4 \pm 17.8$ & $90.5 \pm 19.5$ & $P>0.05$ \\
\hline \multirow[t]{2}{*}{ Normal lipid gp } & $110.8 \pm 18.3$ & $86.2 \pm 15.3$ & $94.5 \pm 18.3$ & $P>0.05$ \\
\hline & $P>0.05$ & $P>0.05$ & $P>0.05$ & \\
\hline \multicolumn{5}{|l|}{ Serum $B_{2}$ microglob. } \\
\hline Dyslepidemic gp & $109.0 \pm 17.4$ & $106.0 \pm 23.6$ & $112.1=11.8$ & $P>0.05$ \\
\hline \multirow[t]{2}{*}{ Normal lipid gp } & $109.8 \pm 10.9$ & $103.3 \pm 16.3$ & $103.2 \pm 16.3$ & $P>0.05$ \\
\hline & $P>0.05$ & $P>0.05$ & $P>0.05$ & \\
\hline \multicolumn{5}{|l|}{ Urinary albumin } \\
\hline Dyslepidemic gp & $4.16=3.15$ & $52.2=28.03$ & $7.76 \pm 4.1$ & $P<0.05$ \\
\hline \multirow[t]{2}{*}{ Normal lipid gp } & $5.04 \pm 2.22$ & $56.7 \pm 21.97$ & $9.1 \pm 4.6$ & $P<0.05$ \\
\hline & $P>0.05$ & $P>0.05$ & $P>0.05$ & \\
\hline \multicolumn{5}{|l|}{ Urinary $\mathrm{B}_{2}$ microglobulin } \\
\hline Dyslepidemic gp & $105.3 \pm 89.7$ & $297.8 \pm 234.9$ & $332.13 \pm 281.6$ & $P<0.05$ \\
\hline \multirow[t]{2}{*}{ Normal lipid gp } & $105.7 \pm 106.7$ & $379.0=408.0$ & $412.86 \pm 364.4$ & $P<0.05$ \\
\hline & $P>0.05$ & $P>0.05$ & $P>0.05$ & \\
\hline
\end{tabular}

Dyslepidemic group comprised 8 patients. Normal lipid group comprised 7 patients. 
Table (10): Correlation between the urinary albumin level and the urinary B2-microglobulin with some echocardiographic and labora... parameters among the thrombolytic group ( $n=15)$.

\begin{tabular}{|l|c|c|c|}
\hline \multirow{2}{*}{ parameters } & $\begin{array}{c}\text { Urinary albumin } \\
\text { level (24hr } \\
\text { value) }\end{array}$ & $\begin{array}{c}\text { Urinary B2-microglobulin } \\
\text { level }\end{array}$ \\
\cline { 3 - 4 } & $\mathrm{2}=+0.540$ & $\mathrm{r}=+0.34$ & $\mathrm{r}=+0.242$ \\
\hline LV-ejection fraction & $\mathrm{P}<0.05$ & $\mathrm{P}>0.05$ & $\mathrm{P}>0.05$ \\
\hline LV-percent fibrous & $\mathrm{r}=+0.637$ & $\mathrm{r}=+0.416$ & $\mathrm{r}=+0.346$ \\
shortening & $\mathrm{P}<0.05$ & $\mathrm{P}>0.05$ & $\mathrm{P}>0.05$ \\
\hline Fasting blood sugar & $\mathrm{r}=+0.243$ & $\mathrm{r}=-0.109$ & $\mathrm{r}=+0.094$ \\
& $\mathrm{P}>0.05$ & $\mathrm{P}>0.05$ & $\mathrm{P}>0.05$ \\
\hline CPK (peak valve) & $\mathrm{r}=0.147$ & $\mathrm{r}=-0.564$ & $\mathrm{r}=+0.014$ \\
& $\mathrm{P}>0.05$ & $\mathrm{P}<0.05$ & $\mathrm{P}>0.05$ \\
\hline LDH (peak value) & $\mathrm{r}=-0.509$ & $\mathrm{r}=-0.565$ & $\mathrm{r}=-0.164$ \\
& $\mathrm{P}<0.05$ & $\mathrm{P}<0.05$ & $\mathrm{P}>0.05$ \\
\hline S. createnin & $\mathrm{r}=+0.104$ & $\mathrm{r}=-0.247$ & $\mathrm{r}=-0.387$ \\
& $\mathrm{P}>0.05$ & $\mathrm{P}>0.05$ & $\mathrm{P}>0.05$ \\
\hline Creatinine clearance & $\mathrm{r}=+0.302$ & $\mathrm{r}=+0.116$ & $\mathrm{r}=+0.059$ \\
& $\mathrm{P}>0.05$ & $\mathrm{P}>0.05$ & $\mathrm{P}>0.05$ \\
\hline
\end{tabular}


432 STUDY OF RENAL FUNCTION AFTER THROMBOLYTIC etc ...

\section{DISCUSSION}

\section{Renal glomerular function:}

The present study showed that use of thrombolytic therapy produced transient glomerular impairment in the form of transient microalbuminuria (table 4 \& Figure 3), although it did not produce any clinical proteinuria

Howeve'r Lynch \& his associates (1995) (10), published that thrombolytic therapy had resulted in clinical proteinurina (urinary protein excretion $>0.15 \mathrm{gm} / 24 \mathrm{~h}$ ). in $82 \%$ of their studied patients. But, their estimation for proteinuria did not include admission sample (as in this study) which could delineate a certain percentage of their studied patients having proteinuria on admission (10). Also, they found proteinuria in $27 \%$ of their studied patient who received no thrombolytic therapy. This later could be attributed to the higher age of their patients (mean 64 years) than in our studied patients (mean 48 years, table 1). Moreover, they did not exclude patients with renal failute.

Lynch \& his associates (1995)(10) had reported also, that proteinuria related to streptokinase therapy had resolved by day 5 after infarction. The microalbuminuria in our studied patients was detected 24 hour after thrombolytic therapy in 12 patients $(80 \%)$ and disappeared completely on the 7 th day of therapy (table 4 \& Fig 3). This means that in both studies it was transient.

The occurrence of microalbuminuria among the different thrombolytic subgroups showed no difference in relation to the presence or absence of diabetes mellitus, hypertension, mild renal impairment as well as the presence of abnormal lipids (table 5).

Analysis of serial absolute values of urinary albumin excretion rate (in $\mu \mathrm{g} / \mathrm{min}$ ) among the whole thrombolytic group (table 4 \& Fig 1) and different thrombolytic subgroups (tables 6-9) showed significant increase in urinary albumin excretion in $1^{\text {st }}$ day after therapy than the admission and the $7^{\text {th }}$ day values $(P<0.05$, table 3$)$. This means that thrombolytic therapy has resulted only in transient microalbuminuria and did not produce clinical proteinurina (all values were less than $150 \mathrm{mg} /$ day), and this effect has no relation to the presence of diabetes mellitus, hypertension, dyslipidemia or mild renal impairment.

The microalbuminuria observed among thrombolytic subgroups was 
not associated with any changes in serum creatinine, creatinine clearance and serum B2-microglobulin. Microalbuminuria can also be associated with an acute phase response, in acute myocardial infarction (27), but the onset and resolution of the microalbuminuria was more rapid than in acute phase response. The small protein load contained in a bolus dose of streptokinase would be unlikely to cause proteinuria as a result of protein load (28). The excess microalbuminuria seen in the thrombolytic group could not be explained on the bases of acute myocardial infarction or its complications and/or differences between the thrombolytic and nonthrombolytic groups as all clinical and laboratory parameters were more or less similar at the start of myocardial infarction. The only difference is the use of streptokinase among the thrombolytic group. The non thrombolytic group on the other hand showed no microalbuminuria (table 3).

The use of intravenous streptokinase in acute myocardial infarction improved left ventricular performance and survival (29). The presence of significantly better left ventricular. performance parameters (ejection frac- tion (EF) and percent fractional fiber shortening (\%FS), table 1) among the thrombolytic group could explain that better thrombolysis and reperfusion improve the left ventricular contractily (and help in wash out of thrombolytic end product into circulation). A significant positive correlation was found between the degree of microalbuminuria and left ventricular systolic functions parameters $(E F, \% F S)(r=$ $0.54 \& 0.64, p<0.05$ respectively) (table 10). From this it could be concluded that, the more successful the thrombolytic therapy and consequently improvement of global left ventricular function, the more is the degree of microalbuminuria. The concomitant increase in thrombolytic end products with efficient thrombolysis could be related to the pathogenesis and degree of transient microalbuminuria.

Microalbuminuria could be explained by complex mediated injury of the renal glomerular basement membrane and immune complex formation after streptokinase therapy in acute myocardial infarction $(30)$ and serum sickness and renal injury (31). At the same time, this microalbuminuria can be explained by renal vascular injury due to cholesterol embolization syndrome (32). 


\section{STUDY OF RENAL FUNCTION AFTER THROMBOLYTIC etc ...}

\section{Renal tubular function :}

Although the urinary albumin excretion which reflect glomerular performance showed transient elevation after thrombolytic therapy, the urinary B2-microglobulin which reflect renal tubular function showed progressive abnormal elevation after thrombolytic therapy as show in (table 4 and Fig 2 \& 3).

The abnormal urinary $\mathrm{B}_{2}$-microglobulin values of $>370 \mu \mathrm{g} / 24 \mathrm{hr}$ was found in 5 patients (33.3\%) receiving streptokinase by the 1st day of therapy and in 7 patients $(44.66 \%$ ) by the 7 th day (table $4, P<0.05$ ). However no abnormal values was found among patients not receiving streptokinase therapy (table $3, P>0.05$ ).

No difference was observed as regard the abnormal urinary B2microglobulin among different thrombolytic subgroups (tables $5-9$ ). This means that diabetes mellitus, mild renal impairment, hypertension or dyslipidemia did not alter the behaviour of tubular function to thrombolytic therapy.

The $\mathrm{B}_{2}$-microglobulin is an endogenously produced substance with a molecular weight low enough to be fil- tered completely across renal glomeruli. Nearly all the fiitered B2microglobulin is actively reabsorbed by renal proximal tubular cells and catabolized during the reabsorptive process in the kidney tubules (33). If the reaicsorption process by the renal tubules is disturbed, the majority of filtered B2-microglobulin (100-200 mg/ day) is excreted in the final urine (34).

The renal removal of low molecular weight protein, B2-microglobulin, from serum encompases three component mechanisms including gomerular filtration, tubular reabsorption and metabolism and excretion of the non reabsorbed protein with urine. Thus the urine concentration of $\mathrm{B2}$ microglobulin is dependent on the amount filtered by the glomeruli and on tubular reabsorption of this protein. Tubular reabsorption of B2microglobulin is normally about $99.9 \%$ of the filtered fraction and only about $0.1 \%$ is excreted in the urine, in which its concentration is maximally about $370 \mu \mathrm{g} / 24 \mathrm{~h}$ (35). Concentration of B2microglobulin depends mostly on integrity of the proximal renal tubules and thus can be used in diagnosis and follow up tubular dysfunction (36).

The elevation of urinary B2- 
microglobulin observed among thrombolytic group can not be explained by neither the increase of its production, (which is endogenously constant), nor by any abnormal glomerular function (as creatinine clearance was within the normal range and the presence of normal serum B2-microglobulin level in whole thromholytic (table 4) and all subgroups (tables 6-9). Thus, the abnormal urinary B2-microglobulin values ( $>370 \mu \mathrm{g} / 24 \mathrm{hr}$ ) found in 5 patients with thrombolytic therapy by $1^{\text {st }}$ day and in 7 patients on the $7^{\text {th }}$ day could be attributed to impaired tubular reabsorption after thrombolytic therapy and this was probably not be related to the hemodynamic changes during acute myocardial infarction as it did not present among any patient not eceving thrombolytic therapy.

The impaired tubular reabsorption after thrombolytic therapy could be explained by the abnormal haemorrheology of blood and formation of immune complexes as well as the massive production of fibrinolytic products after successful lysis of intracoronary microthrombi. Also, cholesterol embolization syndrome may have some role. The increased incidence of $a b$ normal urinary B2-microglobulin on the 7 th than the $1^{\text {st }}$ day after thrombo- lytic therapy (46.6\% versus $33.3 \% \mathrm{P}$ $>0.05$, table 4), although non significant, but may suggest that the impaired tubular reabsorption is related more to immune complex deposition.

Significant negative correlation was found between urinary B2 microalbulin and peak CPK $(r=-0.56, p<$ $0.05)$ and $\mathrm{LDH}(r=-0.57, \mathrm{P}<0.05)$ (table 10). This signifies that the increase in B2-microglobulin was inversily related to infarct size and so the degree of tubular injury after thrombolytic therapy is reversly related to infarct size. This means that B2microglobulin is related to effective thrombolysis; opening of occluded vessel, preserving myocardium and consequently left ventricular performance as well as wash out of the thrombolysis end products which could produce the impaired renal tubular function.

In Conclusion : Thrombolytic therapy has resulted in transient (glomerular dysfunction) in the form of microalbuminuria by the 1 st day after therapy and resolved completely by 7 th day. This microalburninuria was not related to the presence of diabetes mellitus, hypertension, mild renal impairment or dyslipidemia, but 
436 STUDY OF RENAL FUNCTION AFTER THROMBOLYTIC etc ...

correlated positively with the success of thrombolysis and improvement in global systolic left ventricular functions.

Thrombolytic therapy has resulted in abnormal renal tubular function which started by $1^{\text {st }}$ day after therapy and persisted on the $7^{\text {th }}$ day. This abnormal tubular function was not related to the presence of diabetes mellitus, hypertension, mild renal impairment or dyslipidemia, but correlated with effective thrombolysis.

Thromholytic therapy can be used safely (unless there is other contraindication) among patients with diabetes mellitus, hypertension. dyslipidemia as well as the presence of mild renal impairment.

A follow up study to the residual effect of thrombolytic therapy on renal tubular function should be looked for to evaluate the course of this abnormal urinary B2-microglobulin values.

\section{REFERENCES}

1. Fuster V (1992): Mechansim of diseases pathology of CAD $\&$ acute coronary syndrome. $\mathrm{N}$ England $\mathrm{J}$ Medicine, 326:24.

Vol. 32, No. 1 \& 2 Jan. \& April, 2001
2. Willerson JT, Campbell WB, Winniford MD, Schmitz J, Appril P, Firth BG, Ashton J, Smitherman T, Bush L and Buja LM (1984) : Conversion from chronic to acute coronary artery disease speculation regarding mechanisms. Am J Cardiol, 54: 1349.

3. Malek AM, Alper SL, Izumo S (1999) : Haemodynamic shear stress and its role in atherosclerosis. JAMA, 286: 2035-2042.

4. Davies MJ (2000) : The pathophysiology of acute coronary syndromes. Heart, 86: 361-6.

5. Tomoda $H$ (1988) : Atrial natriuretic peptide in acute myocardial infarction. Am J Cardiol, 62: 1122.

6. Pasternak RC, Braunwald $E$ and Sobel BE (1994) : Acute myocardial infarction. In Heart Disease, A textbook of cardiovascular medicine, Braunwald E. (ed); An HBJ International edition, W.B. 
Saunders, London, Chapter 39 p: 1200.

7. Fox KA (2000) : Acute coronary syndrome presentation clini$c a l$ spectrum and manageinent. Heart, 84: 93.

8. McGrath KG, Leffren $B$, Alexander J, Kaplan K and Patterson R (1984) : Allergic reactions to streptokinase consistent with anaphylactic or antigenantibody complex mediated damage. J Allergy Clin Immunol, 73: 453.

9. Lynch M, Pentecost BL, Littler WA, Stockly RA (1994) : Overt and subclinical reactions to streptokinase in acute myocardial infarction. Am J Cardiol, 74: 849.

10. Lynch M, Nancy V, Hoffmann, Constantine N, Aroney (1995) : Thrombolytic treatment and proteinuria. $\mathrm{Br}$ Heart J, 74: 354.

11. Murray C and Alpret JS (1994): Diagnosis of acute myocardial infarction. Curr Opin
Cardiol, 9: 465.

12. Oliver ITA (1955) : Spectrophotmetric method for determination of creatine phosphoskinase and myokinase. Biochem J 61: 116.

13. Chapell JP and Allaf M (1990): Automated quantification of creatine kinase MB isoenzyme in serum by radial partition immunoassay, with use of stratus analyzer. Clin Chem, 36: 99.

14. Nachlas M, Morgulies S, Goldberg $J$ and Setigmam $A$ (1960) : Anal Biochem, 1: 317.

15. Hare C (1950): Proc Soc Exp Biol Med, $74: 148$

16. Osberg I (1990) : Effects of storage time and temperature on measurement of small concentrations of albumin in urine. Clin Chem, 36(8): 1428.

17. Grenner and Dati (1983) : B2microglobulin determination by a new enzymatic immuMANSOURA MEDICAL JOURNAL 
438 STUDY OF RENAL FUNCTION AFTER THROMBOLYTIC etc ...

noassay. Clin Chem 29: 24. Lopes V (1977) : Clin Chem 23: 1240 .

883.

18. Shahangian S, Brown PI and Ash OK (1984): Turbidemetric measurements of total urinary proteins. A revised method. Am J Clin Pathol, 81: 651.

19. Doumas BT, Walson WA and Biggs HG (1971) : Clin Chem Acta, 31: 87.

20. Perry WB, Doamas $B T$ and Bayre PD (1983) : A candidat refernce method for determination of bilirubin in serum. Test for transferability. Clin Chem 29: 297.

21. Reithman $S$ and Frankel $S$ (1957) : Am J Clin Pathol, 28: 56 .

22. Allain CC, Poon LS and Chan (1974) : Enzymatic determination of total serum cholesterol. Clin Chem, 20(4): 470.

23. Fassati $C$ and Prencip A (1982) : Clin Chem, 28: 2077.

Vol. 32, No. 1 \& 2 Jan. \& April, 2001
25. Cooly RN and Schreib $\mathrm{MH}$ (1980) : Textbook of radiology of the heart and great vessels, 3rd (ed). London, Williams and Wiblins, P: 83.

26. Henery WL, Demeria A, Gramiach P, et al (1980) : Report of the American Society of Echocardiography on nomeclature and standards in two dimensional echocardiography. Circ ulation, 62: 212.

27. Kooper IG and Shachman NH (1954) : Level of C-reative protein as a measure of acute myocardial infarction. Proc Soc Exp Biol Med, 86: 95.

28. Schwartz M, Bidani A and Lewis E (1986) : Glomerular epithelial cell structure and function in chronic proteinuria induced by homologus protein-load. Lab Invest, 55: 673.

29. White HD, Nerris RM, Brown 
MA (1987) : Effect of intravenous streptokinase on left ventricular function and survival after acute myocardial infarction. N Engl J Med, 317: 850 .

30. Freysdottir J, Ormarsdottir S and Sigfasson A (1993) : Evaluation of in vivo immune complex formation and complement activation in patients receiving intravenous streptokinase. Clin Exp Immunol, 194: 28

31. Davies KA, Mathieson P, Winearls CG, Rees AJ, Walport MJ (1990) : Serum sickness and acute renal failure after streptokinase therapy for myocardial infarctiol. Clin Exp Immunol, Apr 80(1): 83 .

32. .Wong FK, Chan SK and Inigtz LS (1995) : Acute renal failure after streptokinase therapy in patients with acute myocardial infarction. Am J
Kidney Dis, 26(3): 50

33. Berner GM and Conard NE (1969) : Catabolism of human B2-microglobulin by the rate kidney. Am J Phy'sic!, 217: 1359.

34. Peterson PA, Evrine PE and Berggard I (1969) : Differentiation of glomerular, tubular and normal proteinuria: determination of urinary excretion of B2microglobulin; albumin and total protein. J Clin Invest, 48: 1189

35. Statius VS and Schardijan GHC (1983): B-microglobulin and the renal tubule. In non invasive diagnosis of kidney disease. P: 103-138.

36. Eid HSH (1989) : Diagnostic value of B2-microglobulin in hepatorenal disorders in comparison to standard laboratory tests. M S Thesis, Mansoura University. 
440 STUDY OF RENAL FUNCTION AFTER THROMBOLYTIC etc ...

\title{
دراسة وظائف الكلى بعد الملاج بمذيبات الجلطة في مرضى إحتشاء عضلة القلب الحاد
}

\author{
أ. أ. عصام محمد السيد محفوظ د. إيمان السيد الصفتى

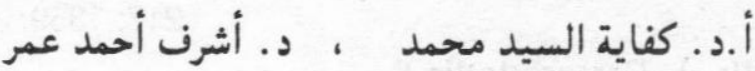 \\ قسم الباطنة العامة والتحاليل الطبية - كلبة طب ا'منصورة
}

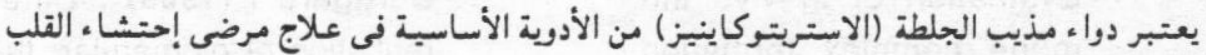

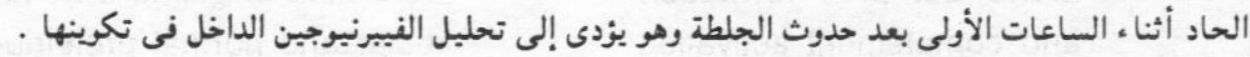

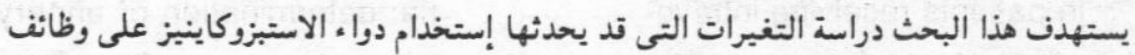

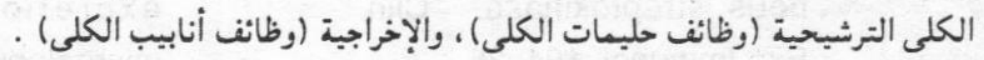

وقد اشتملت هذه الدراسة على عدد ـr مريضاً بعانون من مرضى احتشاء عضلة القلب الحادة بوحدة

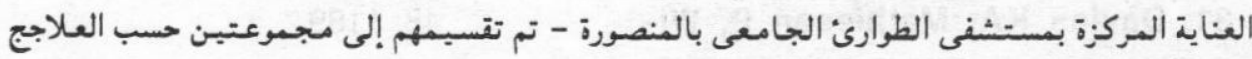

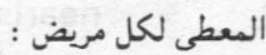

أ) المجموعة الأولى : ومكونة من عدد 10 مريضاً لم يشمل علاجهم إعطاء دواء الاستربتوكاينيز

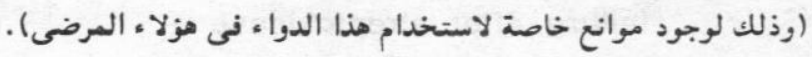

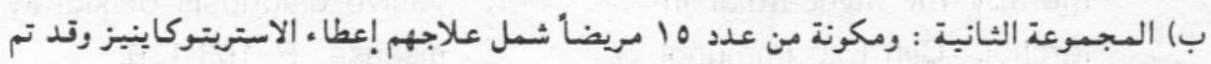

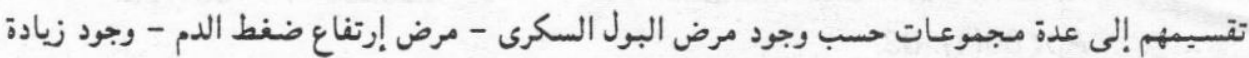

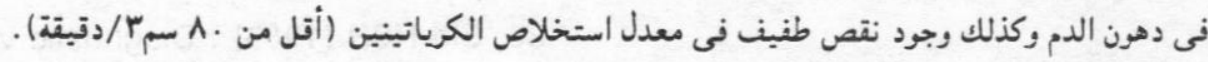

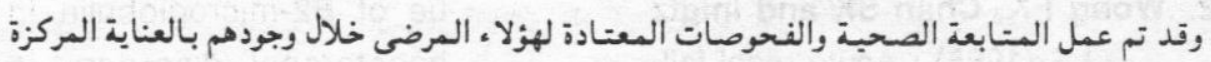

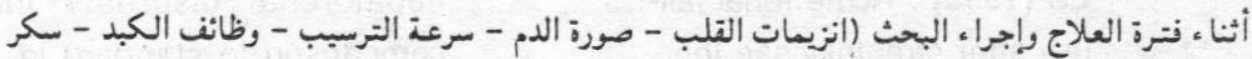

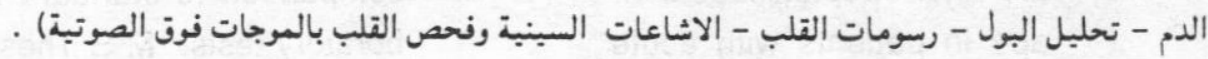

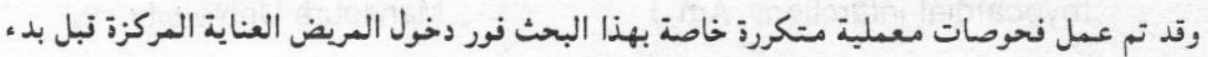
العلاج (اليوم الأول) ، وفى اليوم التالى لأخذ العلاج وكذلك فى البوم السابع من بدء العلاج وشملت : (i) تحاليل معملية لتقيبم وظائف حليمات الكلى (الوظائف الترشبحبة للكلى) وشملت : 
- نسبة إخراج الألبيومبن بالبول (وتعبين وجود المبكرو البيومبنوريا) . (ب) تحالبل معلية لتقيبم وظائف أنابيب الكلى (الوظائف الإخراجبة للكلى) وشملت - مستوى البيتا- - r مبكروجولبيولين بالبول .

$$
\begin{aligned}
& \text { - مستوى البيتا- r مبكروجولبيولبن بالدم. } \\
& \text { رقد أظهرت النتائج مايلى : }
\end{aligned}
$$

أولا : المرضى الذين لم يشـلمل علاجهم إعطا، دواء الاستبرينوكاينبز : لم يوجد هناك أى تغبيبرات معلبة تشبر إلى خلل فى وظائف الكلى أثنا ، نترة العلاج.

$$
\text { ثانيا : المرضى الذين شمل علاجهم إعطا ، دواء الاسترينوكاينيز لوحظ : }
$$

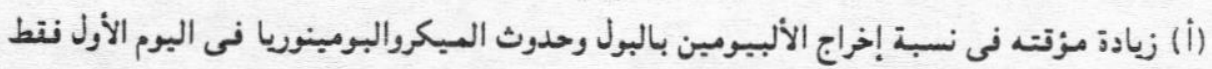

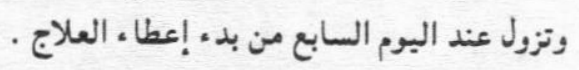

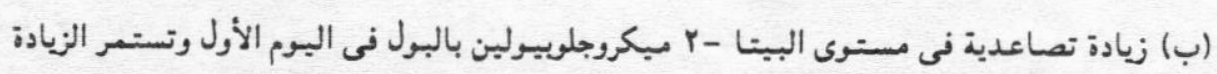

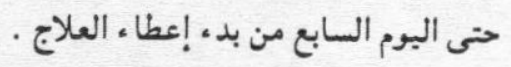

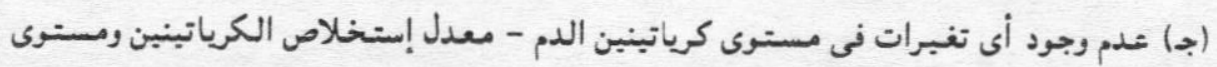

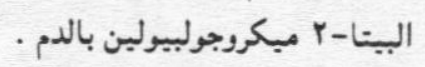

(د) وجود علاقة إرتباطية موجبة بين درجة البكرو البيومين فى البول ورظائف البطين الأيسر .

(هـ) وججود علاقة إرتباطية سالبة بين مستوى البيتا-r ميكروجلوبيولين بالبول انزيمات القلب .

(و)عدم وجود فرق فى التغبرات السعملية التى ظهرت نتبجنة وجود مرض البول السكرى - مرض فرض

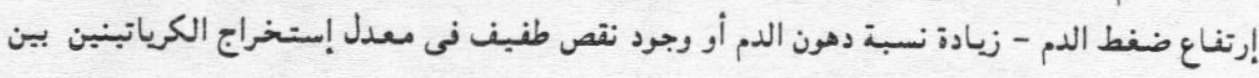

$$
\text { المرضى. (ارع. }
$$

\section{رقد خلصت الاراسة إلى :}

- عمل دراسة ممتدة (متتابعة) لمتابعة التغبرات التى بحدثها إستخدام دواء الاستربتوكاينيز على وظائف أنابيب الكلى . - ملى

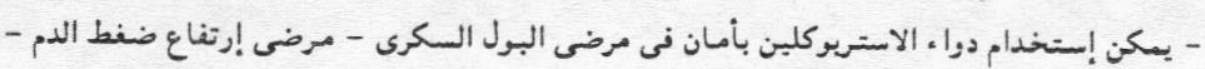

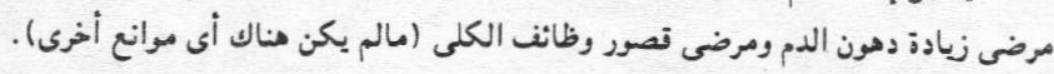


